\title{
Study on the promoting role of dragon boat movement in cultivating college students' team consciousness
}

\author{
Hongchun $\mathrm{Jia}^{1}$, Songbo $\mathrm{He}^{1, *}$, Shiyang Ding ${ }^{2}$, Zhouwei Yao ${ }^{2}$ \\ ${ }^{1}$ Beihua University, Jilin, China \\ ${ }^{2}$ Beihua University, College of physical education, Longteng innovation and entrepreneurship \\ education base \\ *Corresponding author
}

Keywords: Dragon Boat race; team consciousness; promoting role

\begin{abstract}
. the dragon boat is our outstanding traditional sports project, has a long history, rich cultural connotation, the fitness effect is outstanding, especially in recent years, based on the support of the country national traditional sports project, has been developed for the dragon boat sport has a certain influence and welcome the broad masses of Chinese traditional sports, play a positive role the dragon boat culture heritage. At the same time, the Dragon Boat Sport for athletes in the game together, forge ahead in unity and hard work, which coincides with the modern campus culture and school spirit construction goal is consistent, which promotes the rapid development of Dragon Boat Sports Project in Colleges and universities. This paper studies the impact of dragon boat race on team spirit of college students, and reveals the effect and influence of dragon boat training on team spirit of College students.
\end{abstract}

\section{Research Significance}

The team spirit can be summarized as follows: a team of all members of their team satisfaction and recognition, can take the initiative to team interests first, innovative and creative development, give full play to the individual at the same time, to take into account other team members, initiative to collaborate with others, dedication to achieve team interests strive. The dragon boat sport is not a simple paddle can achieve good results, it is twenty people in the same boat, including drummer, helmsman, rower, and some also include Gong hand, achieve the perfect fit, show the Dragon Boat Sport, forge ahead courageously and pull together in times of trouble, make concerted efforts, unity and hard work of the dragon boat, done in one vigorous effort spirit.

We can see that in order to achieve good results, not only the Dragon Boat Dragon Boat Sport all athletes keep consistent, also requires that all athletes with the psychological consistency between athletes to mutual understanding, mutual trust, mutual tolerance, solidarity and cooperation, we have the same goal, do a boat one, in order to get good grades. Therefore, in training, not only to carry out technical, tactical, physical and other aspects of training, but also should pay attention to athletes team spirit training.

\section{Research objects and methods}

\subsection{Study subjects}

Beihua University class 2015 sports education, sports training professional four classes for the study, a total of 120 students.

\section{2.research methods}

\subsection{1 documents and materials law:}

Through the library books, related data and the Dragon Boat Dragon Boat Training and team spirit of the query Chinese journal full text database and the Internet, in order to find out the suitable theoretical basis to provide relevant information for this. Understand the relevant research situation and draw on relevant technical knowledge. 


\subsection{2 experimental method:}

Four classes of grade 2015 in our school were selected, two were selected as experimental class, and two selected as control class. Each class arranges the same class hour quantity, the experimental class arranges the Dragon Boat course, the comparison class arranges the track and field curriculum.

\subsection{3 questionnaire survey}

A total of 120 questionnaires were issued and 120 copies were recovered. All the questionnaires were qualified, and the effective rate of recovery was $100 \%$. The reliability and validity of the questionnaire is 0.85 , and it has higher credibility.

\subsection{4 mathematical statistics:}

SPSS software is used to statistics and process the data.

\subsection{5 interview method:}

Through interviews and discussions of experts, suggestions are made for the formulation of questionnaires and the writing of this paper. In the experimental group, there were $30 \%$ strong cognitive degree, $19 \%$ in general, and $51 \%$ in poor. In the control group, there were $32 \%$ strong cognitive degrees, accounting for $20 \%$, and the poorer ones were $48 \%$. This shows that there are no obvious differences in six aspects: cooperation awareness, cognitive level and cohesion, communication ability, collective honor and trust. It can be concluded that the control group and the experimental group meet the experimental requirements.

\section{Dragon boat movement's influence on team spirit of college students and analysis}

After the experiment, the experimental team had a strong team spirit accounted for 59\%, generally accounted for $24 \%$, the poor accounted for $17 \%$. In the control group, there was a strong team spirit of $31 \%$, accounting for $24 \%$, and the poor accounted for $45 \%$. The experimental research shows that dragon boat sports have significant and good effect on promoting and cultivating team spirit of College students.

College students not only improve their sense of cooperation and cohesion, but also improve their communication ability and trust. This is mainly because the dragon boat sport is to have a goal of the project, at the same time, also must unite together, to cultivate students' strong collective sense of honor and collective sense of belonging in peacetime training, conscious of the individual behavior linked to the team, voluntary sacrifice for the team all the strength.

\section{Effective suggestions}

Experiments have proved that dragon boat movement is one of the effective means to train team spirit. The experimental results also show that dragon boat sports play a very significant role in cohesion, collective honor and cooperative consciousness. At the same time, it plays a very important role in students' communication ability, trust and cognition. If we strengthen the dragon boat movement from the following aspects, it will achieve better results.

\section{1 establish a perfect Dragon Boat course training system.}

At present, the research of dragon boat movement theory lags behind the practice; the construction of the dragon boat sport curriculum system lack of guidance of scientific problems, will directly affect or restrict the Dragon Boat Sport in the dissemination and development of colleges and universities in China and other countries in the world. In this paper, through the analysis of feasibility of Dragon Boat Sports in Colleges and universities of our country, using the basic principle of higher education on curriculum and teaching, according to the national laws and regulations and related policies, combined with the characteristics of dragon boat movement, to the dragon boat sport curriculum system as the research object, the general rules reveal its emergence, formation and development from the macro, preliminary a discussion on how to build the Dragon Boat Sport Curriculum System in China, including its theoretical framework and practice content and subject system and security strategy. Try to put forward to reflect the times, specification and application, the dragon boat sport curriculum system especially highlight the complementary characteristics of Chinese civilization of the Olympic culture, while inheriting traditional culture 
and spirit, realize the all-round development of college students and improving the quality of the target, to cultivate more talents to promote the development of dragon boat, dragon boat movement.

\section{2 strengthen the team spirit construction of College Students}

It is of great significance to cultivate the team spirit of College students. It can not only improve students' sense of collective and cooperation, train their sense of discipline and sense of responsibility, but also play a positive role in shaping the good personality of college students and enhancing their ability to adapt to society. To evaluate the students' team spirit by using fuzzy comprehensive evaluation method, easy to grasp the current development level of education, the lack of analysis, targeted to find the causes, and put forward targeted education strategies.

\section{3 overcome the shortcomings and enhance the competitive level of Dragon Boat Race}

In recent years, the number of colleges and Universities Carrying out Dragon Boat projects has been increasing, and the development momentum is better. It has played a good role in inheriting the outstanding Chinese dragon boat culture among college students. The leaders of most universities pay more attention to dragon boats, and have some incentive policies for coaches and athletes, but they are not strong enough. The vast majority of coaches and athletes believe that the facilities are poor and can not meet the normal training. The age structure of dragon boat coaches in Colleges and universities is mainly middle-aged, with a higher degree of education and bachelor's degree; part-time coaches are the majority; the selection of athletes mainly refers to physical fitness; coaches lack opportunities for training and further education. Dragon boat sport team of men than women, with full-time students, training period in the 2 years following the new heavier proportion, mostly through the selection to participate in dragon boat teams, training to focus on training before the game, most of the teams to train 5 times a week and every time in 3 hours, and every year the number of participants is less, the lack of accumulation of competition experience. Only by raising the level of dragon boat racing can more people participate in this sport.

\section{Acknowledgement}

This paper is a research project of Beihua University students' innovation project, horizontal topi c: Dragon Boat land training equipment (NO:201602014).

\section{Reference}

[1] Wu Guangjin, Qin Tokumasu. [J]. connotation of dragon boat culture and contemporary value of Heilongjiang national series, 2010 (6)

[2] Wang Kaizhen, Hu Juan, Yang Fenghua. Study on the development of the [J] sports culture daokan dragon boat race in China, 2010 (03)

[3] Xifeng Li, Wang Hongyu, Huang Liu. The cultural connotation of dragon boatrace [J] Journal of Qiqihar Teachers College, 2007 (03)

[4] times of spring. Chinese martial arts and cultural heritage studies [D]. Northeast Normal University, 2008.

[5] Yang Jun, Guo Faming, Hao Yanzhen. A cultural morphological analysis of the modernization of dragon boat racing [J]. Journal of Hebei Institute of Physical Education, 2012 (6): 89-91.

[6] He Yingfeng. Analysis of the international promotion of the dragon boat movement in new form [J]. Heilongjiang Institute of interest, 2011 (35): 180.

[7] Zhu Kai. On Wushu cultural inheritance in the international communication of Chinese Wushu [D]. Beijing Sport University, 2010. 